\title{
CORRIGENDUM
}

\section{bring the poor back in! inequalities, welfare and politics}

doi:10.1057/eps.2014.16; published online 23 May 2014

Correction to: european political science (2014) 13, 187-200. doi: 10.1057/eps.2014.4

Since publication of this article, it has been noted that there is an error on page 194 in the following sentence:

'The qualitative survey, conducted among people taken in charge by charities and social services, including non-citizens ( 80 per cent) and non-registered voters ( 30 per cent of the French citizens, more than four times the national average), sheds light on the political attitudes and behaviour of the very poor'.

The correct sentence should read as follows:

'The qualitative survey, conducted among people taken in charge by charities and social services, including non-citizens ( 25 per cent) and non-registered voters ( 30 per cent of the French citizens, more than four times the national average), sheds light on the political attitudes and behaviour of the very poor'. 